La Revue

des Droits

de l'Homme

\section{La Revue des droits de l'homme}

Revue du Centre de recherches et d'études sur les droits fondamentaux

$2 \mid 2012$

Revue des droits de l'homme $-\mathrm{N}^{\circ} 2$

\title{
Les femmes au Sénat
}

M. Berinzon, L. Bonhomme, Laurie Marguet, L. Rebours et M. Sylla

\section{OpenEdition \\ Journals}

Édition électronique

URL : http://journals.openedition.org/revdh/222

DOI : $10.4000 /$ revdh.222

ISSN : 2264-119X

Éditeur

Centre de recherches et d'études sur les droits fondamentaux

Édition imprimée

Date de publication : 1 décembre 2012

Référence électronique

M. Berinzon, L. Bonhomme, Laurie Marguet, L. Rebours et M. Sylla, "Les femmes au Sénat », La Revue des droits de l'homme [En ligne], 2 | 2012, mis en ligne le 28 novembre 2013, consulté le 10 décembre 2020. URL : http://journals.openedition.org/revdh/222 ; DOI : https://doi.org/10.4000/revdh.222

Ce document a été généré automatiquement le 10 décembre 2020.

Tous droits réservés 


\title{
Les femmes au Sénat
}

\author{
M. Berinzon, L. Bonhomme, Laurie Marguet, L. Rebours et M. Sylla
}

1 Si la sous-présence des femmes est un phénomène qui touche tous les domaines de la sphère politique française, c'est au Sénat qu'elle est la plus flagrante. Certes, la part des femmes élues députées est proche de celle des femmes élues sénatrices puisqu'en 1958, elles représentaient 1,6\% des élus à l'Assemblée nationale et 1,9\% au Sénat. Trente ans plus tard, 5,9\% des élus à l'Hémicycle en 1993 sont des femmes, contre $5 \%$ aux élections sénatoriales de $1992^{1}$.

2 Pourtant, il existe une différence majeure entre les deux Chambres concernant l'accès des femmes à un mandat parlementaire : de 1958 à 2000, la sous-présence des femmes au Sénat est la conséquence d'un taux faible de candidatures. En d'autres termes, si les femmes ne comptent que peu d'élues au Sénat, c'est en partie parce qu'elles ne sont qu'une infime proportion à briguer ce mandat. En effet, alors que les candidats aux législatives de 1993 comptent 19,5\% de femmes, celles-ci ne représentent, en 1992, que 9,9\% des candidats au Sénat.

3 L'histoire de la place des femmes au Sénat est d'abord celle d'un paradoxe: alors qu'elles constituent plus de la moitié de l'électorat français, qu'elles ont acquis l'égalité des droits politiques avec l'ordonnance du 21 avril 1944, et que le mouvement féministe est au cœur du débat public dans les années 1960-1970, ces avancées ne se sont pas traduites dans les faits par une progression significative de leur représentation.

4 La période gaulliste se caractérise par une stagnation persistante du faible nombre de sénatrices; jusqu'en 1974, les élues à la Chambre haute ne dépassent pas les $2 \%$. Par ailleurs, on constate qu'une fois admises à participer à la chose publique, les sénatrices sont souvent affectées à des commissions permanentes non valorisées car liées à une représentation sexuée des thématiques. C'est ainsi que lors des premières élections sénatoriales de la $\mathrm{V}^{\mathrm{e}}$ République, quatre des cinq femmes élues sont membres de la commission des affaires sociales ou des affaires culturelles. Au cours de cette première phase de la nouvelle République, le parti communiste fait figure de précurseur et se démarque des autres partis en présentant des listes, sinon paritaires, réservant aux femmes des chances d'être au moins éligibles. 
5 Ce n'est qu'à partir des élections du 22 septembre 1974 que la proportion des élues augmente, faiblement, mais de façon continue pour atteindre $5,9 \%$ en 1998. Il faut dire que le contexte politique de l'époque est particulièrement propice aux changements des mentalités et des pratiques politiques maintenues sous la présidence de Charles De Gaulle. Valéry Giscard d'Estaing, président d'une France qu'il veut moderne, entend faire accéder les femmes à la vie politique en les nommant notamment à des fonctions gouvernementales plus ou moins importantes (il s'agit en majorité de secrétariats d'État ${ }^{2}$.

6 Le Mouvement de libération des femmes (MLF) renouvelle le débat sur la place des femmes dans la société, mais leur présence sur la scène politique reste due au « fait du prince » et peine à être soutenue par le suffrage universel. Plusieurs facteurs sont à l'origine de cette évolution au ralenti. D'une part, il se peut que les efforts du pouvoir exécutif pour rendre plus visible la participation des femmes au niveau gouvernemental relèvent plutôt du symbole et de bas calculs électoraux que d'un véritable engagement pour une juste représentation. D'autre part, les partis politiques ont un rôle indéniable pour permettre aux femmes d'être élues : c'est en effet à eux que revient l'organisation des listes soumises au vote.

7 Bien qu'elles soient de plus en plus nombreuses à siéger au Palais du Luxembourg, cette timide progression ne doit pas éclipser le fait qu'une partie des sénatrices a en fait obtenu un mandat en remplacement d'un sénateur (c'est-à-dire par voie de suppléance). D'autres sont réélues plusieurs années de suite, comme si dans l'esprit des grands électeurs, les sénatrices déjà élues étaient des "perles rares ", exceptions à la règle.

8 Les femmes au Sénat restent donc une exception, un modèle que les partis n'encouragent à suivre que du bout des lèvres. Compte tenu du peu de sièges à pourvoir dans chaque département, et eu égard à la particularité du mode d'élection des sénateurs (suffrage universel indirect), l'exclusion des femmes se trouve institutionnalisée et ne peut être combattue que par une réforme des institutions. Cette idée émerge dans les années 1980, alors que la gauche arrive au pouvoir. C'est sous l'impulsion de François Mitterrand que la promotion des femmes en politique s'amplifie sérieusement, avec entre autre la création d'un ministère des droits des femmes, la nomination d'un Premier ministre féminin (Édith Cresson), et plus généralement la féminisation du corps politique. Pour autant, cette vague féminine n'atteint que marginalement le Sénat, au sein duquel on ne compte toujours que neuf femmes élues en $1986^{3}$.

9 En 1992, elles sont seize à être élues, puis dix-huit en 1995 ; cette multiplication étant une conséquence du débat sur la parité qui anime la société française dans son ensemble. En 1995, le candidat à la présidentielle Jacques Chirac s'empare de la problématique de la «crise de la représentation politique ». Il promet la création d'un observatoire de la parité entre les femmes et les hommes, et fait de ce sujet l'un de ses principaux thèmes de campagne. Si les femmes représentent l'autre moitié de l'humanité, et donc une part non négligeable de l'électorat français, notre régime démocratique doit-il rester inerte face à leur sous représentation? Une fois de plus, le contexte politique a des répercussions directes sur la composition des institutions, y compris au Sénat, même si l'impact y est moins spectaculaire qu'à l'Assemblée nationale (10,9\% d'élues en 1997). 
10 L'histoire de la représentation des femmes au Sénat de 1958 à 2000 illustre à merveille " la monopolisation du pouvoir par un sexe, [ce] mal français par excellence, car prenant appui sur des institutions, celles de la $V^{e}$ République, qui font barrage à l'entrée des femmes en politique $»^{4}$. Au-delà du mode de scrutin propre aux élections sénatoriales, quelles sont les raisons pouvant être avancées pour expliquer la désertion par les femmes de la Haute Assemblée?

11 «En vain prétend-on que l'égalité civile accordée à la femme a pour corollaire nécessaire son émancipation politique. C'est méconnaître absolument le rôle de la femme dans l'humanité. Destinée à la maternité, faite pour la vie de famille, la dignité de sa situation sera d'autant plus grande qu'elle n'ira point la compromettre dans les luttes du forum et dans les hasards de la vie publique. Elle oublierait fatalement ses devoirs de mère et ses devoirs d'épouse si elle abandonnait le foyer pour courir à la tribune. Elle n'y apporterait pas d'ailleurs la modération de langage et la netteté des conceptions, qui sont indispensables dans les usages parlementaires. [...] On a donc parfaitement raison d'exclure de la vie politique les femmes et les personnes qui, par leur peu de maturité d'esprit, ne peuvent prendre une part intelligente à la conduite des affaires publiques $»^{5}$. Bien qu'extraite d'une thèse datant de 1884 , cette citation traduit bien les obstacles auxquels sont confrontées les femmes qui souhaitent accéder à n'importe quel mandat électoral et au mandat sénatorial plus précisément. Trois séries d'explications peuvent être proposées à la situation déplorable de la représentation féminine au Sénat ${ }^{6}$.

12 En premier lieu, les femmes se considèrent indignes d'occuper de telles fonctions. Cette idée préconçue est véhiculée tout au long de la $\mathrm{V}^{\mathrm{e}}$ République non seulement par les femmes elles-mêmes, mais aussi et surtout par l'élite masculine monopolisant la sphère politique. Elles sont jugées incapables d'avoir des compétences comparables à celles des hommes car elles seraient sujettes à des émotions et à des passions que la vie publique ne saurait tolérer.

13 D'autre part, la poursuite d'une carrière politique par une femme est perçue comme un "abandon de foyer", puisqu'il est bien connu qu'une sénatrice doit gérer trois emplois : son mandat d'élue, sa profession et son métier de mère (ou d'épouse). Or, aucun aménagement n'est mis à leur disposition pour leur permettre de jongler entre ces trois statuts. Leur exclusion serait donc liée à la priorité que les femmes doivent accorder à leur foyer.

14 Enfin, les femmes s'excluraient d'elles-mêmes des enceintes politiques et en particulier du Sénat, réputées pour leur dureté et le machisme ambiants. Sous-représentées au sein des partis politiques chargés d'établir les listes des candidats aux élections, les femmes ont encore moins de chance d'être investies par les mâles de leurs clans : «Ils sont juges et parties, [...] à l'UDF vous avez un bureau politique qui donne les investitures : dixsept membres, dix-sept hommes. Bon, qui est-ce qui va défendre les femmes? "»?

15 Ces préjugés étant profondément ancrés dans la culture politique française, il convient de s'en éloigner en réformant les institutions qui relaient largement ces réflexes. C'est ainsi qu'en juin 1999, une réforme constitutionnelle est votée par le Parlement. Désormais, un alinéa est ajouté à l'article 3 de la Constitution, aux termes duquel : « La loi favorise l'égal accès des femmes et des hommes aux mandats électoraux et fonctions électives ». L'article 4, relatif aux partis, dispose quant à lui que: "Ils contribuent à la mise en œuvre du principe énoncé au dernier alinéa de l'article 3 dans les conditions déterminées par la loi ». 
16 Les années 1990 marquent un tournant dans la prise de conscience de la sousreprésentation des femmes en politique et dans la volonté de modifier cette situation par le biais d'outils juridiques, sans toutefois améliorer la situation autant que l'on aurait pu l'espérer (I). Aujourd'hui, les femmes n'ont toujours pas véritablement bénéficié des réformes législatives et constitutionnelles pour franchir en nombre les portes de la Chambre haute et l'on peut se demander si l'évolution du cadre juridique depuis l'an 2000 suffit à limiter l'impact du contexte sociologique (II).

\section{L'évolution du cadre juridique depuis l'an 2000 : bonds et rebonds}

17 Aussi bien au niveau européen qu'au niveau mondial, la France reste l'un des pays dont le taux de représentation des femmes au Sénat demeure trop peu élevé (A). Si des raisons sociologiques peuvent expliquer ce phénomène, c'est aussi le mode d'élection des sénatoriales qui joue sur la parité dans la haute chambre du Parlement (B).

\section{A. Données comparatives}

Les objectifs du Millénaire pour le développement ${ }^{8}$ visant à atteindre $30 \%$ de femmes dans les positions de leadership en 1995 sont loin d'avoir été atteints dans l'Europe d'aujourd'hui. Si l'on compare, au sein de l'Union européenne, le taux moyen de femmes dans les législatures bicamérales avec le taux moyen de femmes dans les législatures monocamérales, celui-ci reste toujours à peu près égal (à environ $22 \%)^{9}$. Il n'y a donc pas de grande différence entre ces deux régimes sur ce point. Au niveau européen, les femmes ne représentent que $18 \%$ des parlementaires ${ }^{10}$. Dans les pays de l'Union où il existe une chambre haute, le taux de femmes oscille entre $2,5 \%$ (en Slovénie) et 36,6\% (en Belgique). En revanche, dans les treize pays concernés, l'on n'en trouve que deux où le président du Sénat est une femme ${ }^{11}$. La France se situe au « milieu », à la huitième place, avec un taux de $22,1 \%$ de sénatrices. Par ailleurs, le nombre de femmes au Sénat, depuis les dernières élections, n'a pas beaucoup évolué dans la plupart des pays de l'Union européenne ${ }^{12}$.

Cependant, on peut trouver, au niveau mondial, quelques pays avec un taux de femmes au Sénat assez important. Ces pays sont éparpillés un peu partout dans le monde. Les quatre pays avec les taux les plus élevés sont la Bolivie, le Burundi, le Sénégal et le Swaziland, où plus de $40 \%$ des élus au Sénat sont des femmes ${ }^{13}$.

Relevons qu'il n'y a pas dans ces pays de très fortes corrélations entre ce pourcentage et la «protection juridique des femmes ». Si l'on étudie la monarchie du Swaziland, il n'y a en effet que très peu de lois protectrices envers les femmes. Par exemple, il n'existe aucune protection contre les violences conjugales ${ }^{14}$; les femmes n'ont pas même le droit d'être propriétaires fonciers ou de contracter des prêts bancaires ${ }^{15}$. De même, en Bolivie, aucune loi ne sanctionne le harcèlement sexuel et le viol conjugal n'est pas reconnu ${ }^{16}$. Pour en revenir au Swaziland, la participation des femmes à la procédure législative est, certes, régie par la Constitution qui impose que sur les vingt sénateurs nommés par le roi, huit soient des femmes ${ }^{17}$, mais celles-ci n'ont en aucun cas la possibilité d'être dans l'exercice de cette fonction "colégislateur », car le Sénat n'a 
qu'un rôle consultatif ${ }^{18}$. Cette participation a donc un effet très limité sur l'intégration des femmes dans la prise de décisions.

21 Par ailleurs, en Bolivie, au Sénégal et au Burundi, il n'existe pas de quota pour la représentation des femmes en politique ${ }^{19}$. Il est dès lors notable de relever qu'au Sénégal, alors même que les femmes composent $40 \%$ du corps sénatorial depuis les dernières élections de $2007^{20}$, ce n'est qu'en 2010 que ce pays a instauré une loi qui régule la parité homme-femme dans la vie politique ${ }^{21}$. Les membres de la Haute Assemblée y sont élus d'une manière indirecte, de façon très similaire au système français. Mais au contraire de la France, où le pourcentage de femmes au Sénat est proche de celui de l'Assemblée nationale (alors qu'il s'agit ici d'une élection directe), le pourcentage de femmes élues à l'Assemblée nationale sénégalaise reste beaucoup plus faible $(18 \%)^{22}$.

Dans ces quatre pays (aux taux de femmes sénatrices les plus élevés au monde), on observe que ce taux n'a pas pour effet de laisser une place plus importante aux femmes dans la vie politique, ni même le vote de lois en faveur des femmes. Cette observation rapportée à la France suggère que l'augmentation du nombre de femmes élues au Sénat ne doit pas être un but en si. Ce "décalage » entre le taux d'élue et l'adoption d'une législation en faveur des femmes pourrait être dû au fait que, contrairement aux pays européens qui ont connu un accroissement du nombre de femmes au Sénat plus lent mais une participation des femmes à la vie militante et politique plus structurelle, les quatre pays évoqués, malgré la rapidité de l'accession des femmes aux mandats électifs, ont un tissu militant et une attention politique à la cause moins influent. En conséquence, malgré le nombre important de femmes au Sénat, il faudra probablement attendre plus longtemps pour voir apparaître une conceptualisation nouvelle des relations entre les sexes ${ }^{23}$. La rapidité de ce changement peut aussi expliquer la raison pour laquelle il n'y a pas encore de lien entre élection politique des femmes et législation existante en faveur des femmes.

Si l'on prend en compte tous les pays du globe où le taux de femmes au Sénat est supérieur à $35 \%$, se rajoutent aux quatre pays précédents : l'Australie, la Belgique, le Belize, le Canada, les Pays-Bas, Rwanda et Sainte-Lucie. En examinant les quatre pays développés qui ont un taux de représentation féminine au Sénat supérieur à $35 \%^{24}$, quelques observations sont possibles.

En Belgique, la participation des femmes est réglementée par une loi de 2002 qui impose l'alternance homme-femme pour les deux premiers noms de la liste ${ }^{25}$. L'effet a été immédiat: juste avant la loi, en 1999, il y avait $30 \%$ d'élues. En 2003, le taux est monté à $37,5 \%^{26}$. En Australie, le système est particulier : il s'agit d'un scrutin à vote unique transférable qui a pour effet de favoriser le taux de femmes élues (alors que l'Irlande et Malte, qui utilisent pourtant la même méthode d'élection, gardent un taux assez bas ; ce qui est probablement dû au fait que l'Irlande et Malte sont de petits pays, ethniquement homogènes et traditionnellement catholiques) ${ }^{27}$. Au Canada ${ }^{28}$ et aux Pays-Bas ${ }^{29}$, il n'y a aucune mesure légale sur la parité. Toutefois, les partis politiques se sont créé des quotas qu'ils s'imposent et suivent d'eux-mêmes ${ }^{30}$. Il n'en demeure pas moins qu'aux Pays-Bas, le nombre de femmes élues reste assez bas pour les mandats "d'échelon inférieur» (c'est-à-dire les mandats locaux, avec seulement $17 \%$ de représentants au niveau municipal et $20 \%$ de maires qui sont de sexe féminin) ${ }^{31}$ ainsi qu'au Sénat ${ }^{32}$. Au contraire, le Canada présente un taux beaucoup plus élevé de femmes au Sénat (nommées par le Premier Ministre) que dans les autres assemblées élues ${ }^{33}$. En 
effet, le but originel de l'institution du Sénat canadien était de représenter équitablement les groupes minoritaires (les amérindiens, les francophones, les femmes, etc... $)^{34}$.

Finalement, on ne rencontre pas systématiquement, dans ces états, de lien entre système de quota et fort taux de représentativité des femmes au Sénat. Il est très difficile de déterminer les lois et les politiques qui peuvent augmenter le pourcentage des femmes dans les sénats. Ainsi, l'Italie, qui était le premier état en Europe à instaurer des quotas légaux de candidatures féminines (en instaurant un quota de $33 \%$ pour les communes et provinces de plus de 15000 habitants et de $25 \%$ pour celles avec un nombre d'habitants inférieur) ${ }^{35}$, est loin d'avoir le taux le plus élevé de femmes $(18,4 \%)^{36}$. De même, si l'Australie a un système très similaire au système irlandais et maltais, dans ces deux derniers états, il existe différentes formes d'entraves à l'élection des femmes ${ }^{37}$.

Se situant dans la moyenne, la France, sans être le plus mauvais exemple, ne fait pas pour autant figure d'élève modèle. Se situant à la $34^{\mathrm{e}}$ place au niveau mondial ${ }^{38}$ en ce qui concerne la représentation des femmes au Parlement (et ce uniquement grâce à une nette amélioration lors des dernières législatives de juin 2012), le pays se hisse entre l'Afghanistan et la Tunisie; il reste de la place pour l'amélioration. Mais en terme de parité, il est important de ne pas se concentrer uniquement sur un pourcentage : comme on a pu le voir avec les pays en voie de développement, augmenter le taux de femmes présentes au Parlement ne suffit pas à améliorer la place des femmes dans la société.

\section{B. Les élections sénatoriales en France : quels outils juridiques pour une meilleure parité ?}

Les sénateurs français sont élus, dès l'âge de $24 \mathrm{ans}^{39}$, par un suffrage indirect ${ }^{40}$, pour un mandat de six ans renouvelable une fois ${ }^{41}$. Leur mandat est incompatible avec celui de député ou de député européen. Environ 145000 personnes participent à ce collège électoral départemental ${ }^{42}$ : des députés, des conseillers régionaux et à $95 \%$ des conseillers municipaux. Depuis le 25 septembre 2011 (dernières élections à ce jour), le renouvellement se fait par moitié tous les trois ans (et non plus par tiers comme auparavant). Depuis le 21 février 2007, cinq sénateurs supplémentaires sont maintenant élus à la Chambre haute ${ }^{43}$, ce qui désormais porte leur nombre à trois cent quarantehuit en France, soit le nombre le plus élevé de l'histoire du Sénat et le maximum de sièges prévu par l'article 24 de la Constitution de $1958^{44}$. S'ajoutant aux cinquantequatre députées déjà élues (ou réélues), vingt-trois nouveaux visages féminins apparaissent alors dans l'hémicycle de la Haute assemblée.

La question du mode de scrutin utilisé lors des élections sénatoriales est importante, car elle est intrinsèquement liée à celle de la parité. Depuis la réforme du 30 juillet 2003, les soixante-dix départements qui élisent trois sénateurs (ou moins) ${ }^{45}$ ont recours à un scrutin majoritaire uninominal ou plurinominal sans vote préférentiel. Les trente départements qui élisent quatre sénateurs (ou plus) ont recours, quant à eux, à un scrutin proportionnel plurinominal ${ }^{46}$. Or, les contraintes paritaires ne concernent que les scrutins proportionnels, ce qui correspond en 2011 à l'élection de $66 \%$ des sénateurs. Ainsi, à la dernière élection, cent douze sénateurs ont été élus à la 
représentation proportionnelle contre cinquante-huit au scrutin majoritaire à deux tours ${ }^{47}$.

Le débat lié à la nécessité d'une parité en politique intervient à la fin des années 1990 lorsque la révision constitutionnelle du 23 juin 1999 introduit l'article 3 de la Constitution $^{48}$. Comme cela a été rappelé, celui-ci autorise la loi (donc le législateur) à " [favoriser] l'égal accès des femmes et des hommes aux mandats électoraux et fonctions électives ». L'article 4 précise pour sa part que les partis politiques contribuent à la mise en œuvre de ce principe dans les conditions déterminées par la loi. La loi du 12 juillet 1999 commence doucement et progressivement la mise en œuvre de la réforme dans le domaine de la politique et crée des délégations parlementaires aux droits des femmes et à l'égalité des chances entre les hommes et les femmes. C'est par la loi du 6 juin $2000^{49}$ - dite "relative à la parité »- que l'étape législative la plus importante en matière de parité a lieu. Elle vise alors à favoriser l'égal accès des hommes et des femmes aux fonctions électives. En effet, celle-ci «constitue en quelque sorte la loi de référence, dans la mesure où elle a mis en place les deux principaux mécanismes sur lesquels repose aujourd'hui la promotion de la parité en politique $»^{50}$.

Dans un premier temps, la loi oblige les partis politiques à présenter autant d'hommes que de femmes pour les scrutins de liste (qui concernent avant tout les élections régionales ainsi qu'une partie des élections municipales et sénatoriales), et prévoit, le cas échéant, une sanction financière. Lorsqu'un parti ou un groupement politique ne respecte pas ses obligations paritaires en vertu de la loi du 6 juin 2000, le montant de la première fraction qui lui est attribué en application des articles 8 et 9 de ladite loi est diminué51. Ainsi, l'écart entre le nombre de candidats de chaque sexe ne peut être supérieur à un, sous peine d'irrecevabilité de la liste.

31 Concernant plus précisément les élections sénatoriales, cette obligation de parité s'impose dans les départements où au moins quatre sièges sont à pourvoir puisqu'il s'agit de ceux dont l'élection procède d'un scrutin proportionnel de liste. Pour les scrutins à un tour, la loi précise que "chaque liste est composée alternativement d'un candidat de chaque sexe $»^{52}$. Pour ceux à deux tours, la loi exige un nombre égal de candidats de chaque sexe figurant «au sein de chaque groupe entier de six candidats dans l'ordre de présentation de la liste ${ }^{53}$.

La loi du 10 juillet $2000^{54}$ est également le signe d'une évolution significative en matière de parité sénatoriale. En effet, celle-ci, en réformant le scrutin proportionnel des élections sénatoriales, introduit alors une obligation stricte de parité entre les hommes et les femmes pour les départements élisant - à l'époque - au moins trois sénateurs. Par le biais de ces nouvelles dispositions, c'est une augmentation de 5,3\% de femmes au Sénat (en passant de 5,3\% à 10,6\%) qui peut alors être constatée entre 1998 et 2001. Ces deux lois de 2000 auront donc produit certains effets positifs puisque vingt-deux sénatrices ont été élues en septembre 2001 parmi les cent deux sièges renouvelés, pour seulement cinq sénatrices sortantes ${ }^{55}$.

33 La réforme du Sénat du 30 juillet 2003 instaure l'actuel mode de scrutin des élections de la Chambre haute. Cette réforme instaure le scrutin proportionnel pour les départements ayant au moins quatre sénateurs (et non plus trois comme avant la réforme). Cette $\operatorname{loi}^{56}$ a fait l'objet d'un examen par le Conseil constitutionnel: les auteurs de la saisine considéraient que ces nouvelles modifications portaient atteintes au principe d'égalité de représentation des citoyens au Sénat. Le Conseil constitutionnel a néanmoins déclaré l'ensemble des dispositions (pourtant régressives) 
de cette loi ${ }^{57}$ comme constitutionnelles ${ }^{58}$. Par conséquent, les départements élisant trois sénateurs (ou moins) sont dorénavant soumis à un scrutin majoritaire, ne comprenant aucune obligation paritaire.

Cette réforme ne va pas dans le sens d'une amélioration de la présence des femmes au Sénat. D'ailleurs, les évolutions constatées en 2004 semblent, dans tous les domaines (élection des femmes, moyenne d'âge, renouvellement), moins importantes que si le scrutin proportionnel avec obligation de présence paritaire de femmes sur les listes avait été maintenu dans ces départements. Le nombre de sièges supplémentaires qui seraient revenus à des femmes est en effet estimé à cinq ${ }^{59}$. L'influence de l'existence ou non d'une obligation de parité, liée au mode de scrutin, sur la présence de femmes dans la Haute Assemblée est ainsi parfaitement visible : en 2001, 7,1\% des femmes sont élues par le biais d'un scrutin majoritaire contre $27 \%$ pour le scrutin proportionnel; en 2004, c'est $4,4 \%$ contre $34,9 \%$ et en $2008,9,5 \%$ contre $27,5 \%$. Malgré tout, une augmentation globale du nombre de femmes présentes dans la Haute Assemblée peut être constatée entre 2001 et 2008 : en 2004, le nombre augmente de 10,6 à 16,9\% (soit $+6,4$ points); et en 2008 , il augmente de $16,9 \%$ à $22 \%$ (soit $+5,1$ points). Cette augmentation constante cesse cependant lors des élections du 25 septembre 2011 qui semblent ainsi marquer un arrêt dans « l'élan paritaire » au Sénat. Alors qu'il y avait $22 \%$ de femmes sénatrices en 2008 , le taux a seulement atteint $22,12 \%$ en 2011 , soit soixante-dix-sept sénatrices sur trois cent quarante-huit. On peut tout de même noter que ce taux reste supérieur à celui de l'Assemblée nationale élue en 2007 (18,72 \%) qui accueille en son sein cent huit femmes, sur un total de cinq cent soixante-dix-sept sièges à pourvoir.

Lors des dernières élections législatives, le 17 juin 2012, cent cinquante-cinq femmes ont été élues députées, pour un total de cinq cent soixante-dix-sept parlementaires. Portant la proportion de femmes élues à $26,9 \%$ soit quarante-huit sièges supplémentaires, il s'agit d'un très net progrès par rapport au scrutin précédent $(18,5 \%$ en 2007). C'est aussi la première fois que l'écart entre le nombre de candidates et d'élues est aussi faible ${ }^{60}$, ce qui signifie à la fois que des femmes ont été investies dans des circonscriptions "gagnables" (et non plus seulement reléguées aux circonscriptions difficiles), mais aussi que la victoire de la gauche permet de voir plus de femmes élues. En effet, les partis de droite ont encore une fois présenté moins de candidates. Il en résulte que, en ce qui concerne les grands partis, on ne trouve que $14 \%$ de femmes dans le groupe UMP (27 sur 188), contre $37 \%$ pour le PS (104 sur 280 ) et $50 \%$ dans le groupe des verts ( 9 sur 18). Plus alarmant, près de quatre cents circonscriptions n'ont jamais élu de femmes alors que seules huit des cinq cent soixante-dix-sept ont toujours élu une représentante.

Juste avant les élections du 17 juin 2012, le nombre de femmes élues au Parlement étaient égal à cent quatre-vingt-cinq pour neuf cent vingt-cinq parlementaires au total, soit un taux de $20 \%$ très exactement. Mais depuis les législatives de 2012, le nombre de femmes élues au Parlement est de deux cent trente-deux pour toujours neuf cent vingtcinq, soit un taux de presque $25 \%$. Avec près d'un quart de parlementaires femmes, la France gagne près de trente-cinq places dans le classement mondial des Parlements les plus paritaires pour se situer dans les quarante premiers. En comprenant un cinquième, puis très récemment un quart de femmes, le Parlement français n'est pas le plus mauvais élève d'Europe en terme de présence féminine, mais il reste très en deçà de la proportion de femmes dans la population française. En effet, chaque recensement 
depuis 1950 a montré que les femmes étaient plus nombreuses que les hommes, soit, en 2007, environ $51,4 \%$ de la population ${ }^{61}$.

Les raisons de cette stagnation sont nombreuses et variées. Sur le plan législatif, les textes ont été peu modifiés : les obligations paritaires du scrutin proportionnel sont toujours bien présentes. Sur le plan législatif, il faut noter que la loi du 31 janvier $2007^{62}$ tendant à promouvoir l'égal accès des femmes et des hommes aux mandats électoraux et fonctions électives est restée parfaitement muette quant à la question de la parité au Sénat. Or, il ne fait aucun doute que cette loi « améliore la parité politique en France. [En effet,] elle s'applique désormais aux membres des exécutifs régionaux et municipaux ainsi qu'à l'élection de l'Assemblée des Français de l'étranger. Pourtant, cette réforme n'est exempte ni d'insuffisances, ni de lacunes, notamment au niveau des assemblées les plus importantes $d u$ pays. L'aggravation des modulations financières lors des élections législatives de même que la création d'un remplaçant pour chaque conseiller général n'auront pas l'efficacité escomptée. Le silence de cette loi sur la parité au Sénat et dans les EPCI [établissements publics de coopération intercommunale] incite même à penser que ces institutions connaîtront prochainement d'autres réformes $»^{63}$.

De plus, comme il l'a déjà été mentionné, la réforme de 2003 a conduit à l'extension du scrutin majoritaire, ce qui a eu pour effet de détériorer certains élans paritaires ${ }^{64}$. Un amendement du sénateur Jean-Louis Masson ${ }^{65}$ avait bien tenté en 2004 de rétablir la représentation proportionnelle dans les départements à trois sénateurs, mais sa tentative a été rejetée à 165 voix contre 161 . Le changement de scrutin dans certains départements n'explique cependant pas tout dans la mesure où il n'influence en réalité que $3 \%$ des sièges ${ }^{66}$.

L'absence d'augmentation de la présence des femmes au Sénat s'explique également par la construction même du système politique. Premièrement, il existe un «cercle vicieux» habituel: "il y a peu de femmes (élues) car il y a peu de femmes (candidates) ». En effet, les candidates au scrutin majoritaire ne sont pas très nombreuses : en 2011, seulement quarante-sept femmes ont été candidates pour deux cent soixante-huit sièges à pourvoir. Peut-être faudra-t-il alors penser à des modalités d'élection encourageant la candidature des femmes dans les scrutins majoritaires... Deuxièmement, les tentatives (masculines) pour contourner les obligations paritaires ne manquent pas: fausses listes dissidentes ${ }^{67}$, tendance des sénateurs à constituer chacun leur propre liste, moyen destiné à n'avoir qu'un seul élu pour les reconduire dans leur mandat. Et ces listes sont pour la plupart conduites par des hommes... Des lors, lorsqu'une seule place est à pourvoir, l'élu sera un homme ${ }^{68}$.

Enfin, l'ineffectivité des sanctions semble également contribuer au ralentissement de l'élection de femmes au Sénat ${ }^{69}$. Si la loi du 6 juin 2000 prévoit bien une sanction financière consistant en une modulation de l'aide publique accordée aux partis politiques en cas de non-respect de la loi ${ }^{70}$, cela reste insuffisant. Le parti socialiste avait déposé une proposition de loi visant à mettre en place des malus pour les partis politiques qui ne respecteraient pas la parité à la fin de l'élection, c'est-à-dire en fonction du nombre d'élues et non pas de candidates. Cette proposition n'a pas été retenue $^{71}$. L'actuel dispositif légal ne s'apparente, ainsi, à tout point de vue, qu'à une obligation de moyen puisque le constituant n'oblige pas les partis à un respect strict de la parité mais les invite à " favoriser $~^{72}$ un égal accès. Selon l'article 1 de la Constitution, on le rappelle, « la loi favorise l'égalité d'accès des hommes et des femmes aux mandats électoraux ». Sur le fondement de cette disposition constitutionnelle, le législateur peut 
alors prendre des mesures incitatives pour promouvoir la parité. Et, on l'a vu, il y a procédé $^{73}$. Mais le cas des élections sénatoriales montre l'effet limité des mesures prévues qui n'ont comme seule finalité que la promotion de la parité lors du choix des candidats.

Cependant, les choses pourraient bien s'améliorer si l'on porte crédit aux récentes déclarations du gouvernement de Jean-Marc Ayrault sur la question. Par ailleurs, après les résultats des dernières législatives, les sanctions ont été sans appel : l'Union pour un mouvement populaire (UMP) perd plus d'un tiers de ses subventions, majoritairement du fait de la perte du nombre d'élus, mais aussi en raison des près de 4 millions d'euros de retenues pour absence de respect des règles sur le taux de présentation des femmes (absence de respect presque assumé par le secrétaire général du parti, Jean-François Copé). Ceci dit, le parti de la nouvelle majorité présidentielle n'est pas en reste avec près d'un million de pénalités; tandis que les autres partis ne souffrent de presque aucune sanction financière ${ }^{74}$.

À cet égard, la ministre des droits des femmes, Najat Vallaud-Belkacem, a annoncé, conformément au programme électoral du nouveau Président, la future suppression des subventions publiques dans leurs intégralités pour les partis ne présentant pas de listes paritaires. La Constitution devra alors non plus seulement favoriser, mais surtout garantir la parité. Afin d'aller dans ce sens, outre la suppression totale des subventions, on pourrait également imaginer des règles de non-cumul de mandat voire une limitation aux nombres de mandats pour chaque élu (comme c'est le cas pour les élections présidentielles). Cela devait permettre, selon les propos de la ministre, de " faire de la parité non plus un luxe mais un automatisme dans la vie politique française ». Cette logique paritaire devrait d'ailleurs être étendue aux municipales, élections qui souffrent encore plus des inégalités de genre.

En l'absence de mesures juridiquement contraignantes, les sanctions qui peuvent être prévues n'ont pas vraiment d'effet dissuasif. Si, comme l'affirmait Jacques Chirac, la parité "est une exigence démocratique et civique qui ne peut plus attendre $»^{75}$, il devient nécessaire d'être plus contraignant. À ce qui n'est qu'une "obligation de moyen ", il conviendrait de substituer une obligation de résultat et des sanctions plus efficaces, comme par exemple, opter pour la suppression totale de toute aide publique pour les partis ne respectant pas la $\operatorname{loi}^{76}$. Pour aboutir à une avancée significative de la paritée ${ }^{77}$, le législateur pourrait aussi uniformiser l'application de la parité à tous les types de scrutin (majoritaire ou proportionnel). Car même s'il est vrai que les partis politiques sont responsables de l'application a minima de la loi, l'incidence des différents modes de scrutins sur l'élection des femmes se révèle assez forte.

Reste maintenant à analyser quelles sont les femmes qui, après être parvenues à être candidates, ont réussi à être élues au Sénat ? Les élues correspondent-elles à un profil type? Le cas échéant, ce profil est-il calqué sur celui du sénateur masculin, homme bourgeois blanc dans la « force de l'âge ».

\section{Le profil des élues actuelles au Sénat : une singularité}

Les données dites sociologiques sur les élues n'étant pas toujours publiques, la plupart des informations ont été obtenues au moyen d'entretiens par courrier électronique ${ }^{78}$. 
Quatre questions - dont une "complémentaire» (à leur discrétion) - leur ont été posées; impliquant une réponse personnelle ${ }^{79}$. Quarante-neuf ont répondu sur les soixante-dix-sept courriels envoyés à l'ensemble des femmes élues au Sénat, soit $53 \%$ des sénatrices ${ }^{80}$. Les développements suivant ne reposent donc pas sur des données exhaustives; mais ils nous semblent, compte tenu des riches informations que l'on trouve sur le site de la Chambre haute (et ce pour toutes les sénatrices) ${ }^{81}$ et d'un nombre de réponses supérieur à la moitié des sénatrices, avoir obtenu un échantillon représentatif ${ }^{82}$.

46 Il en ressort que les élues du Palais du Luxembourg ont un parcours pré-mandat souvent similaire. Cependant, les données sociologiques démontrent que leur profil n'est pas calqué sur celui de leurs homologues masculins (A), même s'il apparaît clairement une distinction « genrée » dans les attributions internes (B).

\section{A. Les caractéristiques sociologiques, données explicatives sur le " parcours du combattant » des élues}

On le sait, les élus du Sénat ont une moyenne d'âge élevée. La grande majorité des élues ont la soixantaine: trente-neuf d'entre elles, soit un peu plus de la moitié, sont sexagénaires. La benjamine a 35 ans tandis que la doyenne aura 80 d'ici peu (et aura donc dépassé l'espérance de vie moyenne des femmes au terme de son mandat). Seulement six d'entre elles ont moins de 45 ans, ce qui s'explique certainement par le fait que l'âge minimal requis pour se porter candidat aux sénatoriales était parmi les plus élevé de toutes les fonctions représentatives, et ce jusqu'en 2011 où il a été abaissé à 24 ans $^{83}$. Sachant que les très jeunes candidats sont considérés comme tels lorsqu'ils ont autour de l'âge minimal requis pour se présenter, une limite d'âge inférieure élevée attire généralement des candidats encore plus âgés. En outre, la longueur du mandat (neuf ans jusqu'en 2011, puis abaissé à six ans) explique le grand âge des sénateurs supposés disposer d'un certain recul dans le jugement. Ainsi, les Sages doivent, selon l'image qui leur est donnée, avoir une certaine expérience de la vie en général et de la vie politique en particulier ; l'âge constitue un facteur identitaire pour l'institution. Les femmes ne constituent donc pas ici une exception : les « jeunes », hommes ou femmes, semblent avoir plus de difficultés à être élus, voire à être candidats.

Cette expérience de la vie politique se vérifie le plus souvent lorsque l'on étudie le parcours des sénatrices. Seulement sept d'entre elles n'ont jamais exercé de mandat avant leur élection au Sénat (et treize n'avaient eu que des mandats municipaux), et toutes celles-ci furent élues après la loi de 2000 sur la parité. Ces professionnelles de la politique ont très souvent été maires ${ }^{84}$, et ont "gravi les échelons »: conseillères générales, conseillères régionales, parfois présidentes ou vice-présidentes de ces conseils, voire députées ou députées européennes (on en trouve 3 anciennes), ou bien déjà sénatrices. À cet égard, un certain nombre d'entre elles cumulent leur mandat de sénatrice avec celui de maire ${ }^{85}$, même si le cumul d'autres mandats locaux est, chez les sénatrices, loin d'être conséquent ${ }^{86}$. Il est intéressant de noter la corrélation entre le nombre d'anciennes maires élues sénatrices et le fait que $95 \%$ des grands électeurs qui les élisent sont des membres de conseils municipaux. À cet égard, la représentativité et l'effet « d'assimilation » entre l'électeur et son élue sont assez prégnants. Nombreuses sont les élues de la Chambre haute qui pensent que leur(s) mandat(s) local(ux) aur(ont) ainsi largement favorisé leur élection au Palais du Luxembourg. 
D'ailleurs, interrogées sur l'influence de la loi de 2000 sur leur élection, nombre de femmes sénatrices tiennent à préciser que c'est leur expérience politique antérieure, voire leur expérience sur leur terrain, qui leur a permis de "faire leurs preuves ». Cette expérience est vue par beaucoup d'entre elles comme le véritable facteur ayant favorisé leur accès au Sénat ${ }^{87}$, et non la loi. Soulignant leur travail personnel et leur engagement, elles voient les lois sur la parité comme une « condition nécessaire mais non suffisante » à l'élection des femmes au Sénat. La grande majorité d'entre elles estiment que si la loi dénote un progrès ${ }^{88}$, il reste encore beaucoup à faire: imposer la proportionnelle dans toutes les circonscriptions ${ }^{89}$ permettrait de la rendre vraiment effective. À ce sujet, on retiendra les propos d'une élue du Luxembourg qui affirme avec véhémence que la «seule vraie élection » est celle du scrutin majoritaire à deux tours. Les candidates confrontées à ce mode d'élection sortent en effet moins souvent victorieuses ; très peu d'entre elles sont d'ailleurs élues en comparaison avec le nombre d'élues par scrutin proportionnel.

En outre, ce mode de scrutin semble favoriser une meilleure diversité des candidats (en dehors du sexe : âge et profession notamment). Plusieurs d'entre elles insistent sur le fait qu'il faudrait, plutôt que changer les textes, faire évoluer les mentalités et pratiques. En effet, et conformément à ce que nous avons évoqué plus haut (voir supra I. B.), certaines déplorent d'elles-mêmes le fait que les partis préfèrent encore payer des indemnités plutôt que de respecter la loi, d'où la nécessité de mettre en place des conditions plus contraignantes. Il semble que seuls les petits partis, par conviction ou parce qu'ils ne peuvent se permettre d'être privés des aides publiques, respectent la loi lors de la présentation de leurs listes. En revanche, la quasi-totalité des femmes communistes soulignent spontanément la volonté avant-gardiste de leur parti de mettre les femmes "en avant ", sans que cela soit un effet d'une norme quelconque. Il est intéressant de relever que quarante-neuf femmes, soit près de $64 \%$ des sénatrices, sont élues en tant que représentantes d'un parti politique de gauche ${ }^{90}$, ce qui permet de faire le lien avec la nouvelle coloration politique du Sénat.

51 Ce qui ressort de ces propos collectés auprès des femmes élues au Sénat est le refus de voir la loi comme le seul «ascenseur électoral » de leur carrière politique. Plus d'une demi-douzaine d'entre elles affirment même catégoriquement que la loi ne les a en rien favorisé et estiment que les femmes devraient pouvoir s'imposer en politique (et être acceptées) sans action législative. C'est notamment le cas pour celles dont la circonscription a changé de mode de scrutin dans un sens défavorable aux femmes (en passant d'un scrutin proportionnel à un scrutin majoritaire, supra I. B.). On peut citer deux exemples : celui d'une sénatrice qui a été "gênée " par cette loi, en la plaçant quatrième de liste derrière trois sortants septuagénaires, et celui d'une autre sénatrice qui s'est vue «imposer la deuxième place sur la liste, alors [qu'elle avait] recueilli $100 \%$ des suffrages de [ses] camarades lors la désignation » du fait que sa « fédération voulait faire élire deux hommes ".

52 Ainsi, « la question de la parité devrait s'inscrire dans un souci plus large de renouvellement des élites politiques: limitation du cumul des mandats dans l'espace et dans le temps, fixation d'un âge limite pour se porter candidat à un siège de parlementaire, etc ».

53 Au niveau professionnel, les sénateurs appartiennent en grande partie à l'élite sociale du pays. Les femmes ne font pas exception. Une petite demi-douzaine d'entre elles dit appartenir à la "classe ouvrière " ou à un "milieu modeste». Ces femmes-là ont la soixantaine et ont quasiment toutes été élues après la loi sur la parité (et souvent 
réélues en 2011). L'une d'entre elles dit appartenir à ce qu'elle appelle la "double minorité »: femme et issue du milieu ouvrier. Mais la plupart se classent dans les couches moyennes (moyennes supérieures) ou supérieures, avec une répartition d'environ 50 \% par catégorie. Un petit nombre d'entre elles précise que leur situation a évolué au cours de leurs carrières professionnelles. Il est évident que leurs indemnités ${ }^{11}$ de fonctions les placent, aux yeux de la majorité des Français, parmi les plus favorisés, et largement parmi les plus fortunés de la société. Mais l'on relèvera que lorsque l'on demande aux sénatrices leur ressenti sur leur appartenance sociale, la plupart répondent davantage en précisant leur formation professionnelle que leur «niveau social », ce qui laisse à penser que le travail et le mérite sont des sentiments forts et revendiqués par les élues du Luxembourg.

Si l'on étudie chacune des professions des femmes élues au Sénat, on peut estimer qu'au moins soixante d'entre elles ont fait des études supérieures. Trois sénatrices étaient attachées parlementaires et six sont juristes de formation; ce qui porte à environ $12 \%$ le taux de femmes ayant pu aborder de près ou de loin le travail de sénatrice en dehors de leurs activités politiques. On note une très forte proportion de femmes issues du corps enseignant, qu'il s'agisse du primaire, du secondaire ou du supérieur. Avec vingttrois femmes concernées, un tiers des sénatrices a l'expérience de l'enseignement (on peut ajouter une éducatrice pour jeunes enfants à ce nombre).

En ce qui concerne les autres caractéristiques des sénatrices, il nous faut relever que seules trois d'entre elles sont ce que l'on appelle parfois des «candidates issues de la diversité ", toutes d'origine nord-africaine. Elles représentent ainsi $3 \%$ des femmes élues au Luxembourg, un taux certainement très inférieur à celui de la population française « issue de l'immigration ». Si l'on inclut leurs trois collègues masculins, moins de $2 \%$ des sénateurs ne sont pas de «type caucasien ». Lorsque l'on parcourt la liste des noms des sénateurs, la particularité de leurs patronymes détonne nettement avec le classicisme de l'état civil des autres sénateurs.

Environ $25 \%$ des sénatrices ont un proche ayant exercé ou exerçant un mandat d'élu. Lorsque c'est le cas, il s'agit souvent de plusieurs membres de la famille ayant été ou étant actifs dans la vie politique, quasiment toujours un ascendant, parfois un conjoint auquel elles peuvent avoir succédé, et un cas de fraternité.

La grande majorité des femmes élues à la Chambre haute sont mères de famille. Seules cinq d'entre elles nous ont indiqué ne pas avoir d'enfants. Majoritairement, elles ont deux enfants; ce qui correspond à la moyenne des Françaises ${ }^{92}$. En proportion, elles sont deux fois moins nombreuses à n'en avoir qu'un mais presque autant à en avoir trois. Rare sont celles qui ont eu une famille plus nombreuse. On relèvera aussi que plusieurs d'entre elles précisent spontanément avoir des petits-enfants, ce qui nous apparaît être un signe d'implication continue dans leurs vies de famille ${ }^{93}$. Il ne nous semble dès lors guère justifié de considérer que l'exercice de hautes fonctions électives soit incompatible avec le fait d'être mère. Au contraire, ces femmes semblent en majorité mener de front vie de famille et vies politique et professionnelle.

\section{B. Au sein du Sénat, des fonctions inégalitaires}

58 En effet, il ne suffit pas d'être élue au Sénat, encore faut-il «se placer» dans les bureaux, les commissions et délégations parlementaires. Les élues soulignent qu'il est souvent difficile de s'imposer non seulement en tant que femme, mais aussi en tant que 
nouvel(le) élu(e). De fait, les mandatures laissent en général peu de place au renouvellement et "les places sont toujours occupées par les mêmes". Quelles fonctions exercent les sénatrices au sein même de la Haute Assemblée?

Le Sénat s'organise de manière interne de sorte d'orienter des groupes de travail sur diverses thématiques. On distingue ainsi les commissions des délégations. Les commissions parlementaires sont des organismes de travail spécialisés dans l'étude de problèmes généraux ou ponctuels avant leur examen en séance publique. Les délégations parlementaires sont eux des groupes permanents d'information ou de contrôle, spécialisés dans un champ d'activité déterminé. Il s'agit donc davantage d'un travail de veille. Dans les deux cas, il s'agit d'avoir des compétences particulières pour ensuite, sur un sujet donné, pouvoir orienter la politique, voire influer sur l'action des gouvernements. On pourrait donc parler "de postes à responsabilité", quant aux directions de ces structures. Or, en ce qui concerne les commissions, seules cinq femmes en sont présidentes et douze en sont vice-présidentes; nombre relativement identique pour les délégations, avec cinq femmes présidentes et sept vice-présidentes. Il convient à ce sujet de préciser que quatre femmes assurent plusieurs fonctions (présidence et/ou vice-présidence) à la fois - commissions et délégations confondues ce qui réduit encore davantage le nombre de femmes à ces postes.

La présidence, quel qu'en soit le type, revient encore une fois majoritairement aux hommes. Toutefois, si trente-deux femmes (soit presque la moitié des élues), n'assurent aucune fonction de présidence (ou de vice-présidence) au sein de la Haute Assemblée, cela ne semble pas de prime abord discriminant puisque environ la moitié des sénateurs n'en assurent pas non plus. Mais il faut rappeler que les hommes sont bien plus nombreux (271 contre 77). Si, comme nous l'avons vu (voir supra en Introduction), la plupart des femmes se voit presque systématiquement attribuées des "responsabilités de type féminin », cela semble moins le cas au Sénat. L'on relèvera notamment le fait que le poste de rapporteur général du budget - poste très important et réputé «exceptionnel» pour une femme - a été récemment occupé par une sénatrice ${ }^{94}$.

61 Le fait que trois femmes ${ }^{95}$ issues du Sénat aient récemment fait leur entrée au gouvernement de Jean-Marc Ayrault démontre une certaine "reconnaissance » et confiance envers les élus du Luxembourg et spécifiquement envers les élus de sexe féminin. La volonté du nouveau Président de la République ${ }^{96}$ de composer un gouvernement strictement paritaire aura sans doute favorisé cette " ouverture », mais cela reste tout de même la première fois qu'un nombre aussi important d'élues de la Haute Chambre se voit proposer de gouverner. Le point positif est que ces nominations n'auront que très légèrement modifié la composition du Sénat, puisque sur trois remplaçants concernés, deux sont en fait des remplaçantes ${ }^{97}$.

62 Autre fonction notable, on notera que sur huit postes, une femme est vice-présidente du Sénat. Quant au Bureau, organe directeur du Sénat et composé du président, des vice-présidents, des questeurs et des secrétaires, il regroupe vingt-six sénateurs au total. Dans sa composition, la proportion des femmes y est un peu meilleure $(27 \%)$ qu'au sein de la Chambre elle-même, mais l'on ne peut s'empêcher de relever que la majorité de ses membres féminins se trouve affectée aux postes de secrétaires.

63 Il est absolument remarquable de noter que seulement quatre femmes ont été élues sénatrices avant 2001. Bien que la loi de 2000 ne semble pas avoir été le seul catalyseur (voir supra II. A.), elle représente tout de même un "point de départ temporel » à 
l'élection de presque toutes les sénatrices. Pourtant, l'on compte nombre de sénateurs qui en sont à leur énième mandat. Les bons résultats en terme de parité lors des dernières élections législatives laissent espérer que la progression partiaire au sein de l'autre Chambre du Parlement, qui stagnait au cours des derniers renouvellements, sera plus significative en 2014 (date des prochaines sénatoriales). Si l'élan se poursuit de la même manière, la parité parfaite devrait être acquise d'ici quatorze années. En outre, le fait que quarante-sept des sénatrices actuelles en soient à leur premier mandat qu'elles aient été élues avant 2011 ou lors des dernières élections - montre que la porte s'ouvre aux "primo-accédants ", a fortiori de sexe féminin. Cela est néanmoins nuancé par le fait que six des élues ont d'abord occupé cette fonction en remplacement (suite au décès ou la démission d'un élu).

Il semble que le "parcours du combattant " auquel sont confrontées les femmes aux ambitions sénatoriales ne s'arrête pas à l'entrée du Palais. Une fois ses portes franchies, les disparités entre sénateurs et sénatrices demeurent: peu de fonctions importantes sont à la charge des élues. Il semble néanmoins que la réalisation de certains efforts, même si essentiellement réalisés récemment, permettent un accroissement de la visibilité des femmes au Sénat, tant par leur nombre que par leurs attributions.

Depuis la loi sur la parité, le nombre de femmes au Sénat a plus que triplé. Cependant, l'accroissement du taux de sénatrices a stagné lors des dernières élections et l'on ne peut que déplorer que le cinquième de femmes élu à la Haute Assemblée ne fasse de la France un meilleur exemple en terme de représentativité des femmes au Sénat.

Afin d'améliorer la démocratie paritaire, deux niveaux d'action paraissent possibles. On peut soit agir par la loi et imposer aux partis un quota ou un pourcentage de femmes aux fonctions électives, soit s'en remettre aux partis politiques eux-mêmes, sous la pression des militants, des sympathisants et de l'opinion publique. Il semble que dans le premier cas, les partis restent souvent pris au dépourvu, comme si «trouver des femmes " se révélait difficile. En effet, le bilan des candidatures semble montrer que sans mesure contraignante, l'élection des femmes ne pourra pas progresser. Dans les départements avec scrutin majoritaire, il y a eu en effet soixante-dix femmes candidates et soixante-cinq d'entre elles (soit 92,9\%) avaient un suppléant homme. En revanche, on comptait cent quatre-vingt-un hommes candidats et seulement cinquante et un d'entre eux avaient une suppléante (soit $28,2 \%$ ). Dans les départements avec scrutin proportionnel, on doit aussi regretter que sur un total de cent trente listes, il n'y en ait eu que vingt-et-une (soit 16,2\%) conduites par des femmes.

Le principal argument avancé est que les femmes font d'autres choix de carrière. Le fait que la moitié de la base électorale ne soit pas attirée par l'offre de participer activement à la vie politique semble pour le moins curieux. Il reste que le « choix » des femmes candidates se fait toujours par les hommes, ancrés de longue date dans les partis et que, comme le souligne une sénatrice, «le fait pour un homme de placer une femme tête de liste ou de défendre la parité fait toujours de lui un saint homme, comme s'il était extraordinaire, incroyablement bon et courageux de promouvoir pareille folie ».

68 Le résultat des élections de 2011 démontre bien que les obstacles à la parité restent divers et nombreux. Les mentalités et la construction du système politique sont 
difficiles à changer et constituent un obstacle à une égale représentation hommesfemmes en politique, alors même qu'« accepter la logique paritaire implique de transposer le principe de l'égalité des sexes dans la pratique politique, en lien direct avec la Constitution et la démocratie $e^{98} "$.

\section{BIBLIOGRAPHIE}

\section{Textes de lois}

Loi n 88-227 du 11 mars 1988 relative à la transparence financière de la vie politique.

Loi constitutionnelle $n^{\circ}$ 99-569 du 8 juillet 1999 relative à l'égalité entre les femmes et les hommes.

Loi $\mathrm{n}^{\circ}$ 2000-493 du 6 juin 2000 tendant à favoriser l'égal accès des femmes et des hommes aux mandats électoraux et fonctions électives.

Loi $n^{\circ} 2000-41$ du 10 juillet 2000 relative à l'élection des sénateurs.

Loi n 2003-327 du 11 avril 2003 relative à l'élection des conseillers régionaux et des représentants au Parlement européen ainsi qu'à l'aide publique aux partis politiques.

Loi $n^{\circ}$ 2003-697 du 30 juillet 2003 portant réforme de l'élection des sénateurs, complétée par la loi $n^{\circ}$ 2007-224 du 21 février 2007 portant dispositions statutaires et institutionnelles relatives à l'outre-mer.

Proposition de loi $\mathrm{n}^{\circ} 505$ déposée au Sénat le 19 septembre 2005 par le sénateur J.-L. Masson tendant à rétablir le scrutin proportionnel avec obligation de parité dans les départements élisant trois sénateurs.

Loi organique $\mathrm{n}^{\circ}$ 2005-1562 du 15 décembre 2005 modifiant les dates des renouvellements du Sénat.

Loi n 2010-11 du 28 mai 2010 instituant la parité absolue homme/femme.

Loi organique $n^{\circ}$ 2011-410 du 14 avril 2011 relative à l'élection des députés et sénateurs.

Décret $n^{\circ}$ 2011-530 du 17 mai 2011 portant convocation des collèges électoraux pour l'élection des sénateurs.

\section{Doctrine-articles}

Brunet C., «Proposition de loi pour pénaliser les partis qui font élire trop peu de femmes », Le Monde, 27 avril 2010.

Carbet L., « Senate Reform : What does Bill C-20 mean for women? », http://

politicalscience.dal.ca/Files/Bio_files/L_Carbert/Senate_chapter.pdf.

Cool J., « Women in Parliament », Library of Parliament, 2010, http://www.parl.gc.ca/content/lop/ researchpublications/prb0562-e.htm. 
Germain J., «L'amélioration partielle de la parité politique par la loi du 31 janvier 2007 : des avancées utiles qui peuvent régler des problèmes urgents ", AJDA, 2007.

Lechevet A., « Le Sénat n’a pas basculé vers la parité », Le Monde, 26 septembre 2001.

Scwindt-Bayer L. A., « Making Quotas Work : The Effect of Gender Quota Laws on the Election of Women », Legislative Studies Quarterly, 2009.

Scwindt-Bayer L. A., Malecki M. et Crisp B. F., « Candidate Gender and Electoral Success in Single Transferable Vote Systems », British Journal of Political Science, 2010, p. 706.

Senac-Slawinski R., « Des quotas légaux et partisans à la parité : panorama des stratégies en Europe », Informations sociales, $\mathrm{n}^{\circ} 151,2009 / 1$.

Sineau M., «Les femmes politiques sous la $\mathrm{V}^{\mathrm{e}}$ République. À la recherche d'une légitimité électorale ", Pouvoirs, n 82, 1997, p. 55.

\section{Ouvrages}

Delre A., Femmes et partis politiques en Italie et en Europe : la démocratie au masculin, Paris, L'Harmattan, 2011.

Morlot E., De la capacité électorale, Thèse, Université de Paris, 1884.

Pionchon S. et Dervielle G., Les Femmes et la politique, Grenoble, Presses universitaires de Grenoble, 2004.

Sineau M., Parité. Le Conseil de l'Europe et la participation des femmes à la vie politique, Strasbourg, Éditions du Conseil de l'Europe, 2004.

\section{Rapports et Colloques}

Halimi G., La Parité dans la vie politique, Rapport de la Commission pour la parité entre les femmes et les hommes, Observatoire de la parité entre les femmes et les hommes, 1999.

Joannin P., «L'Europe, une chance pour la femme », Notes de la Fondation Robert-Schuman, Fondation Robert Schuman, 2000.

Organisation des Nations Unies, « Objectifs du Millénaire pour le Développement », Rapport de 2010.

Sénat français, Rapport n 552, Rapport d'information 2009/2010.

Roudy Y., La Parité bafouée, Actes du Colloque Floirac,27-28 août 2002.

Zimmerman M.-J., Les Effets directs et indirects de la loi du 6 juin 2000, Rapport de l'Observatoire de la parité entre les femmes et les hommes, La documentation française, mars 2005.

\section{Institutions et Organisations internationales (Sources internet)}

Direction de la communication du Sénat, « Le scrutin du 25 septembre 2011, données essentielles », Sénatoriales 2011

http://www.senat.fr/fileadmin/Fichiers/Images/evenement/senatoriales2011/brochure/projet/ Senatoriales-le-scrutin-du-25-septembre-2011.pdf

Inter-Parliamentary Union, Women in national parliaments, 30 novembre 2011 www.ipu.org/wmn-e/classif-arc.htm 
Inter-Parliamentary Union, SÉNÉGAL - Sénat, 17 janvier 2011

www.ipu.org/parline-f/reports/1362_A.htm

ONU FEMMES, Le Progrès des femmes dans le monde : en quête de justice, 2011

http://progress.unwomen.org/pdfs/FR_Report-Progress.pdf

The Electoral Institute of Southern Africa, Election Update - SWAZILAND, 2003

http://dspace.cigilibrary.org/jspui/bitstream/123456789/30402/1/eu_200402sw.pdf?1

\section{NOTES}

1. Ministère de l'Intérieur et Observatoire des inégalités in Les femmes élues au Sénat, E. JacquierLaforge, Mémoire de master 2, Université Paris II Panthéon-Assas, 2002, 133 p.

2. De sexe féminin, on compte onze secrétaires d'État et six ministres au total (pour les gouvernements Jacques Chirac et Raymond Barre I, II et III).

3. D'après les données disponibles sur le site du Sénat: http://www.senat.fr/ancienssenateurs-5eme-republique/index.html

4. M. Sineau, "Les Femmes politiques sous la $\mathrm{V}^{\mathrm{e}}$ République. À la recherche d'une légitimité électorale ", Pouvoirs, $n^{\circ}$ 82, 1997, p. 55.

5. E. Morlot, De la capacité électorale, Thèse, Université de Paris, 1884 (extrait consultable sur le site de l'Assemblée nationale: http://www.assemblee-nationale.fr/histoire/femmes/ citoyennete_politique_anthologie.asp).

6. G. Halimi, «La Parité dans la vie politique », Rapport de la Commission pour la parité entre les femmes et les hommes, Observatoire de la parité entre les femmes et les hommes, 1999, pp. 13-21. 7. S. Pionchon et G. Derville, Les Femmes et la Politique, Presses universitaires de Grenoble, 2004, p. 75.

8. Organisation des Nations Unies (ONU), Objectifs du Millénaire pour le développement, Rapport de 2010, p. 25.

9. Inter-Parliamentary Union, Women in national parliaments, 30 novembre 2011 : http:// www.ipu.org/wmn-e/classif.htm

10. M. Sineau, Parité - Le Conseil de l'Europe et la participation des femmes à la vie politique, Éditions du Conseil de l'Europe, 2004, p. 7.

11. Le Royaume Uni et la Belgique.

12. Par exemple, en Belgique, le taux est passé de $41 \%$ à $38 \%$ depuis les dernières élections : http://ec.europa.eu/social/main.jsp?langId=fr\&catId=89\&newsId=854\&furtherNews=yes

13. Inter-Parliamentary Union, Women in national parliaments, op. cit.

14. ONU FEMMES, Le progrès des femmes dans le monde: En quête de justice, 2011, p. 13 : http:// progress.unwomen.org/pdfs/FR_Report-Progress.pdf

15. The Electoral Institute of Southern Africa, Election Update - SWAZILAND, 2003, p. 30 : http:// dspace.cigilibrary.org/jspui/bitstream/123456789/30402/1/eu_200402sw.pdf?1

16. ONU FEMMES, op. cit., p. 135.

17. En plus de ces vingt sénateurs, dix sont nommées par l'Assemblée nationale.

18. The Electoral Institute of Southern Africa, Election Update - SWAZILAND, op. cit., p. 31.

19. ONU FEMMES, op. cit., pp. 123-124.

20. Inter-Parliamentary Union, SÉNÉGAL - Sénat, 17 janvier 2011: www.ipu.org/parline-e/ reports/2362_B.htm

21. Loi n 2010-11 du 28 mai 2010 : http://www.jo.gouv.sn/spip.php?article8213

22. Inter-Parliamentary Union, SÉNÉGAL - Sénat, 17 janvier 2011, op. cit.

23. J. Cool, «Women in Parliament», Library of Parliament 2010: http://www.equalvoice.ca/ canadian_reports.cfm 
24. Le Canada, l'Australie, la Belgique et les Pays-Bas.

25. M. Sineau, op. cit, p. 67 : «De plus, la Constitution a été révisée pour préciser, à l'article 10, que 'l'égalité des femmes et des hommes doit être garantie'».

26. P. Joannin, «L'Europe, une chance pour la femme ", Notes de la Fondation Robert Schuman, Paris, Fondation Robert Schuman, 2000, p. $37:$ http://www.senat.fr/uip/Fichiers_pdf/note22.pdf 27. L. Carbet, Senate Reform: What does Bill C-20 mean for women?: politicalscience. dal. ca/Files/ Bio_files/L_Carbert/Senate_chapter.pdf

28. Par exemple, sous la direction de Stéphan Dion, le parti libéral s'est imposé un quota de $30 \%$. 29. J. Cool, op. cit.

30. Il faut noter qu'en Pologne, il y a un système très similaire avec l'Union du travail et l'Union de la liberté qui ont imposé un quota. Cependant, le taux des femmes au sénat polonais n'est que de $13 \%$.

31. Notamment au niveau municipal: http://www.un.org/News/fr-press/docs/2006/ FEM1541.doc.htm

32. Ibidem

33. L. Cabret, op. cit.

34. Ibidem.

35. R. Senac-Slawinski, « Des quotas légaux et partisans à la parité : panorama des stratégies en Europe ", Informations sociales, 2009/1, n 151 ; voir aussi : L. A. Schwindt-Baye, Making Quotas Work: The Effect of Gender Quota Laws on the Election of Women, Legislative Studies Quarterly, 2009.

36. Inter-Parliamentary Union, Women in national parliaments, novembre 2011: http:// www.ipu.org/wmn-e/classif.htm

37. L. A. Schwindt-Baye, M. Malecki et B. F Crisp, "Candidate Gender and Electoral Success in Single Transferable Vote Systems », British Journal of Political Science, 2010, p. 706.

38. « Nombre de femmes à l'Assemblée nationale : la France entre l'Afghanistan et la Tunisie », La Voix $\mathrm{du}$ Nord, article paru le 18 juin 2012 : http://www.lavoixdunord.fr/pour-elles/nombre-defemmes-a-l-assemblee-nationale-la-france-ia2251b0n 523825

39. Loi n 2011-410 du 14 avril 2011.

40. Article 24 de la Constitution française du 4 octobre 1958.

41. Loi organique $\mathrm{n}^{\circ}$ 2005-1562 du 15 décembre 2005 modifiant les dates des renouvellements du Sénat.

42. Pour plus de détails sur le collège électoral, voir le décret n 2011-530 du 17 mai 2011 portant convocation des collèges électoraux pour l'élection des sénateurs (publié au Journal officiel de la République Française ${ }^{\circ} 0115$ du 18 mai 2011, p. 8598, texte $\left.n^{\circ} 17\right)$.

43. Réforme du 30 juillet 2003 complétée par celle du 21 février 2007, $n^{\circ}$ 2007-224 (publiée au JORF $\mathrm{n}^{\circ} 45 \mathrm{du} 22$ février 2007).

44. Texte de la Constitution du 4 octobre 1958 à jour de la révision constitutionnelle du 23 juillet 2008, article 24 : «Le Sénat, dont le nombre de membres ne peut excéder trois cent quarante-huit, est élu au suffrage indirect. Il assure la représentation des collectivités territoriales de la République ».

45. Cela correspond à cent quatre-vingt sénateurs, soit $52^{\circ} \%$ de la Haute Assemblée.

46. Cela correspond à cent soixante-six sénateurs, soit $48^{\circ} \%$ de la Haute Assemblée.

47. Direction de la communication du Sénat, «Le scrutin du 25 septembre 2011-données essentielles ", Sénatoriales 2011: http://www.senat.fr/fileadmin/Fichiers/Images/evenement/ senatoriales2011/brochure/index.htm (dernier accès le 4 novembre 2012).

48. Loi constitutionnelle $\mathrm{n}^{\circ} 99-569$ du 8 juillet 1999 relative à l'égalité entre les femmes et les hommes.

49. Loi $n^{\circ} 2000-493$ du 6 juin 2000 tendant à favoriser l'égal accès des femmes et des hommes aux mandats électoraux et fonctions électives (JORF du 7 juin 2000).

50. Sénat français, Rapport du Sénat $n^{\circ} 552$, rapport d'information 2009/2010. 
51. Article 9-1 de la loi $n^{\circ}$ 88-27 du 11 mars 1988 relative à la transparence financière de la vie politique : "Lorsque, pour un parti ou un groupement politique, l'écart entre le nombre de candidats de chaque sexe ayant déclaré se rattacher à ce parti ou groupement, lors du dernier renouvellement général de l'Assemblée nationale, conformément au deuxième alinéa de l'article 9, dépasse $2 \%$ du nombre total de ces candidats, le montant de la première fraction qui lui est attribué en application des articles 8 et 9 est diminué d'un pourcentage égal à la moitié de cet écart rapporté au nombre total de ces candidats ».

52. Article 3 de la loi $n^{\circ} 2000-493$ du 6 juin 2000, JORF $n^{\circ} 131$ du 7 juin 2000.

53. Article 2, alinéa 1 de la loi $n^{\circ} 2000-493$ du 6 juin 2000, JORF nº 131 du 7 juin 2000.

54. Loi $n^{\circ} 2000-641 \mathrm{du} 10$ juillet 2000 relative à l'élection des sénateurs.

55. Observatoire de la parité, Rapport sur les effets directs et indirects de la loi du 6 juin 2000 de M.J. Zimmerman (rapporteuse générale documentation française), mars 2005.

56. Loi organique du 30 juillet 2003 portant réforme de la durée du mandat et de l'âge d'éligibilité des sénateurs ainsi que de la composition du Sénat.

57. À l'exception de l'article 7 - relatif aux bulletins de vote - de cette loi qui fut déclarée non conforme à la Constitution.

58. Conseil constitutionnel, décision $n^{\circ} 2003-475$ du 24 juillet 2003.

59. Observatoire de la parité, rapport sur les effets directs et indirects de la loi du 6 juin 2000, op. cit.

60. $40^{\circ} \%$ de candidates pour $26,6^{\circ} \%$ d'élues. Voir l'observatoire des inégalités: http:// www.inegalites.fr/spip.php?article59\&id_mot=92

61. Source de l'Institut national de la statistique et des études économiques (INSEE) :

http://www.recensement.insee.fr/tableauxDetailles.action?

zoneSearchField=FRANCE\&codeZone=1-FE\&idTheme=12\&idTableauDetaille=50\&niveauDetail=1

62. Loi $n^{\circ}$ 2007-128 du 31 janvier 2007 tendant à promouvoir l'égal accès des femmes et des hommes aux mandats électoraux et fonctions électives.

63. J. Germain, «L'amélioration partielle de la parité politique par la loi du 31 janvier 2007 : des avancées utiles qui peuvent régler des problèmes urgents ", AJDA 2007, p. 733.

64. Ibidem. L'absence de mesure paritaire dans la réforme de 2003 et l'extension du scrutin majoritaire ont eu pour effet de détériorer la parité. De plus, comme il l'a été mentionné plus haut, la réforme de 2003 aurait fait perdre cinq sièges aux femmes selon l'observatoire de la parité.

65. Proposition de loi du sénateur J.-L. Masson tendant à rétablir le scrutin proportionnel avec obligation de parité dans les départements élisant trois sénateurs, proposition n 505 (2004-2005) déposée au Sénat le 19 septembre 2005.

66. J. Germain, op. cit.

67. A. Lechenet, «Le Sénat n'a pas basculé vers la parité », Le Monde, 26 septembre 2001.

68. Ibidem.

69. Ce n'est cependant pas le cas dans tous les pays du monde, puisqu'au Canada, par exemple, certains partis politiques s'imposent d'eux-mêmes la parité au Sénat (voir supra).

70. Loi $n^{\circ} 88-227$ du 11 mars 1988 relative à la transparence financière de la vie politique, article 9-1 modifié par loi n 2003-327 du 11 avril 2003 - art. 35 JORF 12 avril 2003 : « Lorsque, pour un parti ou un groupement politique, l'écart entre le nombre de candidats de chaque sexe ayant déclaré se rattacher à ce parti ou groupement, lors du dernier renouvellement général de l'Assemblée nationale, conformément au deuxième alinéa de l'article 9, dépasse $2 \%$ du nombre total de ces candidats, le montant de la première fraction qui lui est attribué en application des articles 8 et 9 est diminué d'un pourcentage égal à la moitié de cet écart rapporté au nombre total de ces candidats. Cette diminution n'est pas applicable aux partis et groupements politiques ayant présenté des candidats exclusivement outre-mer lorsque l'écart entre le nombre de candidats de chaque sexe qui s'y sont rattachés n'est pas supérieur à un ». 
71. C. Brunet, «Proposition de loi pour pénaliser les partis qui font élire trop peu de femmes », Le Monde, 27 avril 2010 : http://chantalbrunel.net/2010/04/27/article-du-monde-une-propositionde-loi-pour-penaliser-les-partis-qui-font-elire-trop-peu-de-femmes-la-depute-chantal/

72. Souligné par nous.

73. Y. Roudy, La Parité bafouée, Actes de Colloque, Floirac, 27 et 28 août 2002.

74. S. Laurent, "L'UMP amputée de presque un tiers de son financement ", Le Monde, 19 juin 2012 : http://www.lemonde.fr/politique/article/2012/06/19/l-ump-perd-50-de-sonfinancement-public_1720639_823448.html

75. Propos de l'ancien président Jacques Chirac lors des vœux des assemblées (Assemblée nationale, Sénat, Conseil économique et social) le 3 janvier 2006.

76. C. Brunet, op. cit.

77. En ce sens, voir la proposition de loi du sénateur J.-L. Masson, op. cit.

78. Le site du Sénat donne accès à des renseignements sur chacun des sénateurs. Ceux-ci sont classés par groupe (par département, par commission, par catégories professionnelles, par groupe politique et un groupe est d'ailleurs consacré aux femmes élues). En plus du nom bien sûr, ces indications renseignent nombre d'éléments qui nous ont servi pour notre étude, dont : la date de naissance, la profession, la date d'élection, l'appartenance politique, les mandats locaux et autres fonctions exercées, les fonctions antérieures... et une adresse mail, grâce à laquelle nous sommes entrés en contact avec les élues. http://www.senat.fr/senateurs/femsen.html

79. 1) Avez-vous un parent proche ayant accompli ou accomplissant un mandat électoral ? (si oui, quel est votre de lien de parenté avec cette personne ?) ; 2) Avez-vous des enfants? (si oui, combien ?) ; 3) À quel « niveau social » estimez-vous appartenir? (le but n'étant évidemment pas ici d'établir une grille des « revenus pré-mandat » ni de « catégoriser » nos élues mais d'avoir le ressenti des sénatrices sur leur milieu social d'origine pour étudier l'impact de données purement sociologiques sur l'accès aux hautes fonctions électives) ; 4) Question complémentaire : pensez-vous que la loi sur la parité de 2000 ait favorisé votre élection? (si oui, dans quelle mesure ?)

80. Notre analyse est donc fondée sur des sources brutes. Nos données sont néanmoins anonymisées, non personnalisées, ayant précisé aux destinataires de notre enquête qu'elles ne seraient pas nommément citées. Si certaines ont toutefois précisé qu'elles acceptaient de l'être, nous avons choisi de ne rien en faire par souci d'harmonisation.

81. www.senat.fr/

82. Nous adressons nos très sincères remerciements à toutes les élues ayant répondu à notre sollicitation, et plus particulièrement à celles qui ont accepté de répondre une deuxième fois à notre courriel lorsque la question 2 fut ajoutée à notre étude (voir note supra) et également à celles avec qui nous avons pu nous entretenir directement au téléphone. Nous leur savons particulièrement gré de la rapidité de certaines de leurs réponses, et de l'amabilité et intérêt manifeste de leur très grande majorité.

83. 35 ans jusqu'en 2004 puis 30 ans entre 2004 et 2011.

84. Sur soixante-dix-sept sénatrices, quarante et une exercent toujours, en parallèle de leurs fonctions au Sénat, un mandat municipal (vingt-deux sont maires, huit sont conseillères municipales et onze adjointes).

85. Voir supra (note 72).

86. Aucune sénatrice n'est présidente de conseil général ou régional. Cependant, neuf d'entre elles sont conseillères régionales (dont trois vice-présidentes) et douze sont conseillères générales (dont deux vice-présidentes). À noter que parmi celles-ci, l'une est à la fois viceprésidente de conseil général et vice-présidente de conseil régional.

87. Si l'expérience de la candidate est souvent plus conséquente que celle du candidat, l'accès au Sénat nécessite presque toujours une expérience préalable, bien que l'on observe, chez les hommes et les femmes nouvellement élus, de plus en plus de «nouveau-nés de la politique ». 
88. Certaines soulignent que ladite loi a été davantage bénéfique pour leur élection à des fonctions locales que pour leur élection au Sénat.

89. Seules $52 \%$ des circonscriptions élisent leurs sénatrices à la proportionnelle, c'est-à-dire que la loi sur la parité ne joue qu'une fois sur deux. Les femmes élues au scrutin majoritaire se comptent sur les dix doigts de la main, alors que ce n'est pas le nombre de femmes candidates, à ce type de scrutin, qui manquent.

90. On compte vingt-et-une femmes UMP (Union pour un mouvement populaire) et sept de l'Union centriste.

91. $7100,15 €$ au $1^{\text {er }}$ février 2012 (moins les retenues, soit un revenu net estimé à 5 388,71€).

92. Les femmes françaises ont un taux de fécondité autour de deux depuis les deux dernières décennies. Source INSEE : http://www.insee.fr/fr/themes/document.asp?ref_id=ip1385

93. Sur les réponses que nous avons reçues, neuf d'entre elles ont un enfant, dix-huit d'entre elles en ont deux tandis que sept en ont trois. De façon non surprenante, seules deux sénatrices ont quatre enfants et une seule en a davantage, c'est-à-dire cinq.

94. La nomination récente au gouvernement Ayrault de l'élue en question a eu pour conséquence l'interruption de son mandat. Si nous savons que sa remplaçante est également de sexe féminin (ne modifiant ainsi donc pas la composition " genrée » du Sénat), il n'est pas certain que le poste de rapporteur général du budget échoue de nouveau à une femme.

95. En sus de Nicole Bricq, sénatrice de Seine-et-Marne depuis 2004 et déjà nommée au gouvernement Ayrault, Hélène Conway-Mouret, sénatrice des Français de l'étranger depuis septembre 2011 ainsi qu'Anne-Marie Escoffier, sénatrice de l'Aveyron depuis septembre 2008, ont rejoint le gouvernement lors du remaniement du 21 juin 2012.

96. François Hollande, élu le 6 mai 2012 septième Président de la $V^{\mathrm{e}}$ République française.

97. Les trois remplaçants en question sont, comme leurs titulaires, membres d'un parti de gauche. En l'occurrence, les deux remplaçantes sont membres d'Europe Écologie-Les Verts, ce qui vient grossir les rangs du groupe de ce parti politique au Sénat. Par ailleurs, l'une d'entre elles viendra s'ajouter au nombre "d'élues de la diversité » tout en permettant de laisser inchangé le nombre d'enseignantes élues, étant elle-même professeur de lycée (la seconde remplaçante est, elle, avocate de profession).

98. A. DELRE, Femmes et partis politiques en Italie et en Europe: la démocratie au masculin, Paris, L'Harmattan, 2011, p. 112.

\section{AUTEURS}

\section{BERINZON}

Étudiant du Master 2 Droits de l'homme, Université Paris-Ouest Nanterre La Défense, promotion 2011-2012

\section{BONHOMME}

Étudiant du Master 2 Droits de l'homme, Université Paris-Ouest Nanterre La Défense, promotion 2011-2012 


\section{LAURIE MARGUET}

Étudiant du Master 2 Droits de l'homme, Université Paris-Ouest Nanterre La Défense, promotion 2011-2012

\section{REBOURS}

Étudiant du Master 2 Droits de l'homme, Université Paris-Ouest Nanterre La Défense, promotion 2011-2012

\section{SYLLA}

Étudiant du Master 2 Droits de l'homme, Université Paris-Ouest Nanterre La Défense, promotion 2011-2012 